\title{
Therapie einer Distalbisslage mit mandibulärer Schwenkung - ein Fallbericht
}

\section{Therapy of Distal Occlusion with Mandibular Deviation - A Case Report}

\author{
Autoren \\ Katrin Kley, Britta A. Jung \\ Institut \\ Klinik für Kieferorthopädie, Universität Freiburg \\ Schlüsselwörter \\ Klasse II, Herbstscharnier, mandibuläre Schwenkung \\ Key Words \\ Class II malocclusion, Herbst Appliance, \\ mandibular deviation \\ Bibliografie \\ Inf Orthod Kieferorthop 2020; 52: 267-273 \\ DOI 10.1055/a-1241-9930 \\ ISSN 0020-0336 \\ (c) 2020. Thieme. All rights reserved. \\ Georg Thieme Verlag KG, Rüdigerstraße 14, \\ 70469 Stuttgart, Germany
}

\author{
Korrespondenzadresse \\ Katrin Kley \\ Klinik für Kieferorthopädie \\ Universität Freiburg \\ Hugstetter Straße 55 \\ 79106 Freiburg \\ Deutschland \\ katrin.kley@uniklinik-freiburg.de
}

\section{ZUSAMMENFASSUNG}

Der vorliegende Artikel beschreibt die Therapie eines jugendlichen Patienten mit einer skelettalen Klasse II und mandibulärer Schwenkung mittels funktionskieferorthopädischer Geräte (herausnehmbar und festsitzende Klasse-II-Mechanik).

\section{ABSTRACT}

This article describes the therapy of an adolescent patient with a skeletal class II and mandibular deviation using functional orthodontic appliances (removable and fixed).

\section{Einleitung}

Die skelettale Klasse II ist die in Mitteleuropa am häufigsten vorkommende Malokklusion. Die Prävalenz wird in der Literatur mit 12 bis $34 \%$ angegeben [1-6]. Ätiologisch spielen sowohl exogene $[7,8]$ als auch endogene $[9,10]$ Faktoren eine bedeutsame Rolle. In der überwiegenden Mehrzahl der Fälle liegt die Ursache in einer mandibulären Retrognathie. Da die mandibuläre Retrognathie in der täglichen zahnärztlichen Routine nicht immer leicht zu identifizieren ist, wird ein nicht unerheblicher Anteil der Jugendlichen meist spät in einer kieferorthopädischen Sprechstunde vorgestellt. Dies stellt den Kieferorthopäden regelmäßig vor eine große Herausforderung, da der Erfolg und die Effektivität der Behandlung eng mit der Wachstumskonfiguration und dem zum Zeitpunkt der Behandlung noch verfügbaren Wachstumspotenzial der Patienten gekoppelt sind. Weitere Faktoren, die in diesem Zusammenhang eine Rolle spielen, sind unter anderem der jeweilige Ausprägungsgrad der vorliegenden Dysgnathie, die individuelle Reaktion der parodontalen und skelettalen Strukturen, der Allgemeinzustand des Gebisses, die Patientenmitarbeit (Compliance) sowie Wünsche und Erwartungen von Patienten und Eltern (unter anderem Erwartungen an die dentofaziale Ästhetik).

Zur Behandlung einer skelettalen Klasse-II-Malokklusion können in Abhängigkeit des verfügbaren Wachstumspotenzials bzw. des skelettalen Alters sowohl herausnehmbare als auch festsitzende funktionskieferorthopädische Apparaturen eingesetzt werden $[11,12]$. Während das Tragen von herausnehmbaren funktionskieferorthopädischen Apparaturen besonders effektiv im Wechselgebiss sowie im frühen adulten Gebiss während des pubertären Wachstumsspurts $[13,14]$ ist, nutzen feste Apparaturen wie bspw. die Herbst-Apparatur das Restwachstum aus und ermöglichen damit, Rücklagen des Unterkiefers auch nach dem pubertären Wachstumsschub zu korrigieren. Studien der Arbeitsgruppe Ruf und Bock [14] belegen besonders eindrucksvoll den Erfolg dieser Geräte.

Der vorliegende Patientenfall beschreibt die Therapie eines jugendlichen Patienten mit einer skelettalen Klasse II und einer mandibulären Schwenkung, die sowohl mittels einer herausnehmba- 
ren funktionskieferorthopädischen Apparatur als auch mit einem gegossenen Herbstschanier behandelt wurden.

\section{Fallbericht}

Der Patient ( $>$ Abb. 1) stellte sich im Alter von 11 Jahren in der Hochschulambulanz für Kieferorthopädie vor. Es bestanden keine allgemeinanamnestischen Besonderheiten bzw. Einschränkungen für eine kieferorthopädische Behandlung, die Mundhygiene war als gut zu bewerten. Das Hauptanliegen des Patienten und der Eltern war die Gewährleistung des Durchbruchs der bleibenden Zähne sowie eine Verbesserung der dentofazialen Ästhetik. Zu Behandlungsbeginn befand sich der Patient am Anfang der 2. Wechselgebissphase. Der allgemeine Zahndurchbruch war leicht verspätet. Der Zahn 55 war durch eine unterminierende Resorption ausgehend von 16 vorzeitig verloren gegangen.

Im Oberkiefer lag zudem ein frontaler Engstand sowie Vorwanderungen der Seitenzähne beidseits (1.Q > 2.Q) bei einer Arch Length Discrepancy (ALD) von -5,1 mm vor. Neben diversen Dreh- und Kippständen wies der Oberkiefer ein transversales Defizit von -2 mm auf.

Der Unterkiefer zeigte trotz einem Engstand der Unterkieferfront eine annähernd ausgeglichene ALD von -0,3 mm (berechnet mit Moyers). Der Zahn 41 stand in einer Supraposition.

Die Profilfeldanalyse nach A.M. Schwarz ergab ein nach hinten schiefes Durchschnittsgesicht ( $\mathbf{A b b}$. 1c). Die Gesichtsproportionen waren harmonisch. Das En-Face-Foto des lachenden Patienten zeigte eine positive Lachlinie ( $\triangleright$ Abb. 1b) und eine leichte Abweichung des Kinns nach rechts ( $\triangleright$ Abb. $\mathbf{1 a} \mathbf{a}-\mathbf{b})$.

\section{Diagnose der Okklusion und Bisslage}

Angle-Klasse-II/2-Malokklusion mit seitenungleicher Distalverzahnung ( $\triangleright$ Abb. 1d-h) im Bereich der 6er rechts $1 \frac{1}{4}$ Pb distal, im Bereich der 3er rechst $1 / 4 \mathrm{~Pb}$ distal, im Bereich der Ger links $1 / 2$ Pb distal und im Bereich der 3er links neutral. Nicht abgestützter tiefer Biss, mit vergrößertem Overjet + $3 \mathrm{~mm}$ und Overbite + $5 \mathrm{~mm}$. Der Unterkiefer war skelettal um $2 \mathrm{~mm}$ nach rechts abgewichen.
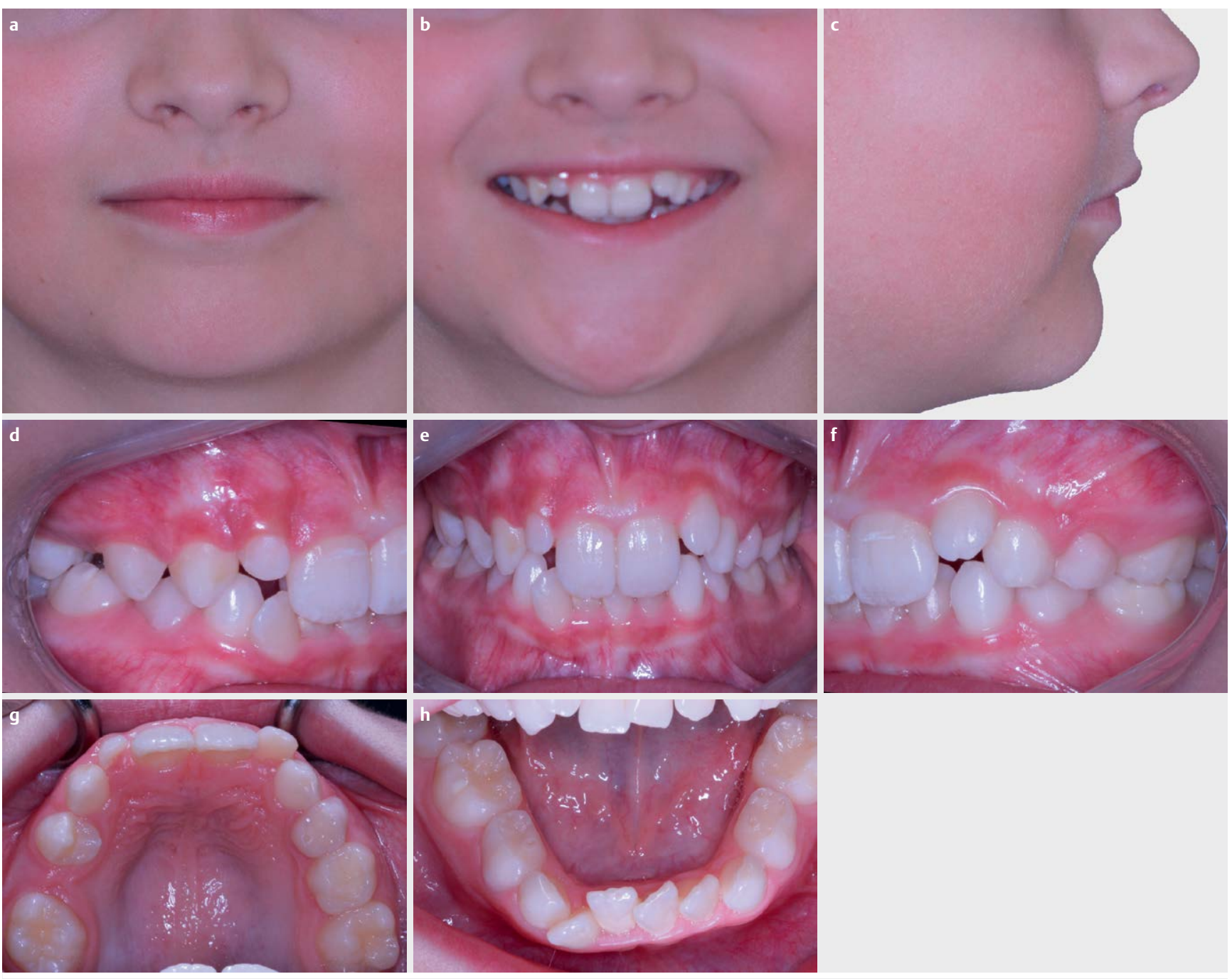

Abb. 1 Klinischer Ausgangsbefund eines 11 Jahre alten Patienten: der Patient befand sich am Anfang der 2. Wechselgebissphase a extraoral frontal, b extraoral frontal lachend, $\mathbf{c}$ Profilansicht sowie $\mathbf{~ d - h}$ die intraorale Ausgangssituation. Der allgemeine Zahndurchbruch war leicht verspätet. 

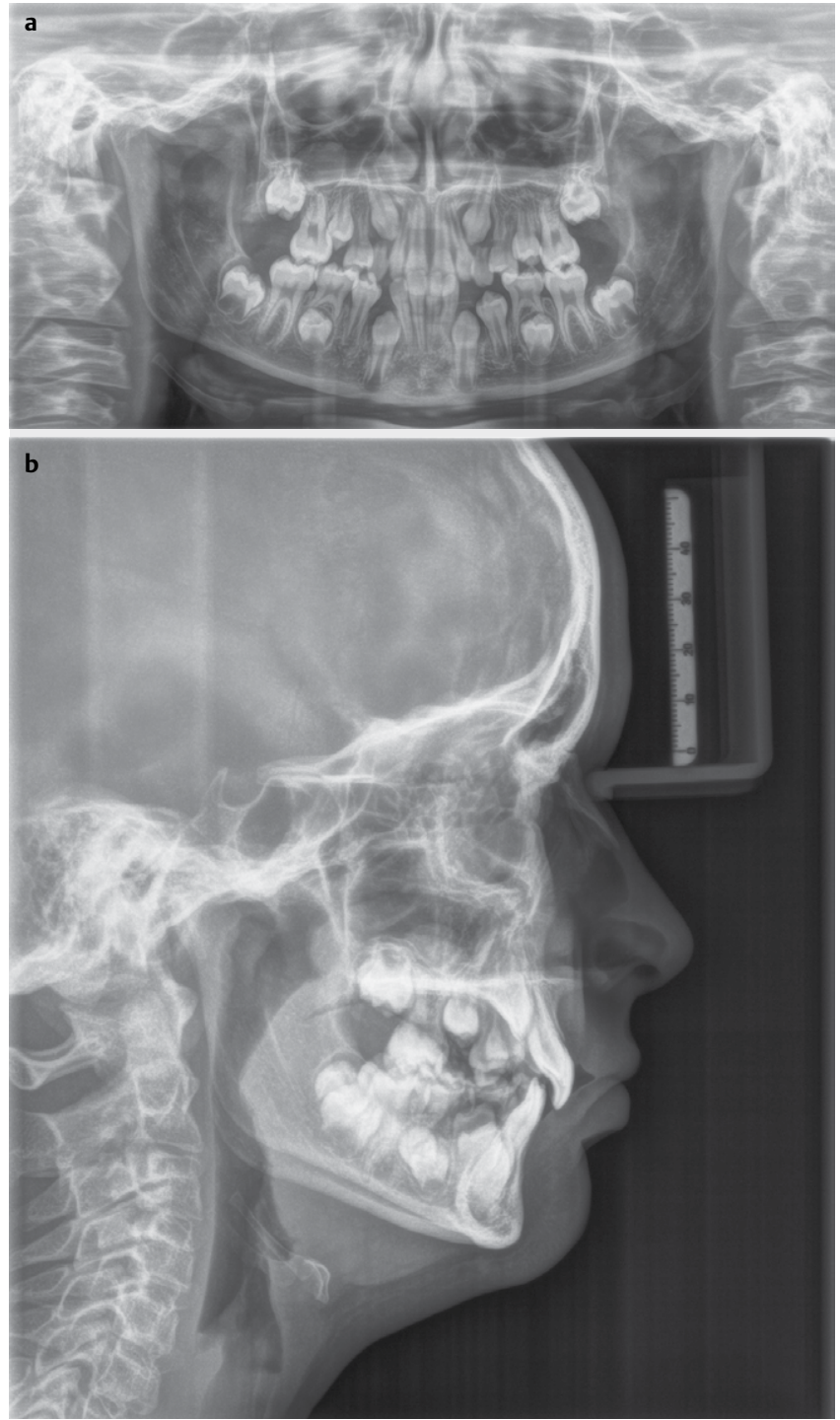

- Abb. 2 Radiologischer Ausgangsbefund: a Panoramaschichtaufnahme und $\mathbf{b}$ Fernröntgenseitenbild vor der Therapie. Alle permanenten Zähne waren angelegt. Die Anlagen der Weisheitszähne 18, 28,38 und 48 waren radiologisch noch nicht sichtbar.

\section{Radiologischer Befund}

Es waren alle permanenten Zähne 17-47 angelegt. Die Anlagen der Zähne 18, 28, 38 und 48 waren zu diesem Zeitpunkt radiologisch nicht nachweisbar ( $>$ Abb. 2a). Eine Verlagerungstendenz und Retention der Zähne 13 und 23 war zu erkennen. Des Weiteren lag eine skelettale Abweichung des Unterkiefers um 3 mm nach rechts vor.

Die Fernröntgenseitenbildanalyse ( $\mathbf{A} \mathbf{b} \mathbf{b} . \mathbf{2} \mathbf{b})$ zeigte eine Distalbisslage mit orthognather Maxilla und leicht retrognather Mandibula bei horizontalem Wachstumsmuster und neutraler Wachstumstendenz. Der Nasolabialwinkel war vergrößert, die Frontzähne waren bimaxillär orthoinkliniert.

\section{Behandlungssystematik und -therapie}

Es wurden im Rahmen der Therapie folgende Therapieziele definiert:

- Ausgleich und Korrektur des transversalen Defizits

- Distalisation der Molaren im Oberkiefer

- Halten des Leewayspaces im Unterkiefer

- Überwachung und Begleitung des Zahnwechsels, insbesondere der Einstellung der Zähne 13, 23, 33 und 43

- Einstellen einer Neutralbisslage

- Korrektur der mandibulären Schwenkung

- Ausformen der Zahnbögen mit Korrektur von Dreh- und Kippständen

- Retentionsmaßnahmen

Vor Therapiebeginn wurden die Zähne 73 und 83 extrahiert. Die Behandlung startete mit einer Vorschubdoppelplatte nach Sander. Das transversale Defizit konnte erfolgreich durch wöchentliche Aktivierung der medianen Dehnschraube im Oberkiefer ausgeglichen werden. Anschließend wurden die Zähne 16 und 26 mittels Aktivierung der Schrauben distalisiert.

Die Zähne 13 und 23 wurden während dieser Phase regelmäßig palpiert und die Mobilität von 53 und 63 überwacht. Ein Jahr nach Behandlungsbeginn hatten sich die Oberkiefer-3er eingestellt.

Die Reevaluation nach ca. 2-jähriger Funktionskieferorthopädie (FKO-Therapie) ( $>$ Abb. $\mathbf{3}$ und 4) ergab im Oberkiefer ein ALD von -2 und $-1,5$ mm im Unterkiefer. Die transversale Breite war ausgeglichen. Die Verzahnung hatte sich rechts auf eine $3 / 4 \mathrm{~Pb}$ distal und links auf eine Neutralverzahnung im Bereich der 6er verbessert ( $\triangleright$ Abb. 3d-h). Der Overjet war durch die Proklination der Oberkieferfront auf $+4,5 \mathrm{~mm}$ leicht vergrößert, der Oberbite auf $+4 \mathrm{~mm}$ reduziert. Es bestand nach wie vor die skelettale mandibuläre Mittelinienabweichung im Unterkiefer um 2 mm nach rechts. Die Fernröntgenseitenbildanalyse ergab eine skelettale Klasse I bei horizontalem Wachstumsmuster.

Zur Korrektur der skelettalen Mittelinienabweichung im Unterkiefer und Einstellung einer Neutralokklusion sowie Reduzierung des Overjets wurde zu diesem Zeitpunkt bei dem 13,8-jährigen Patienten eine gegossene Herbstapparatur eingesetzt. Die Konstruktionsbissnahme erfolgte mit einer Überkorrektur von ca. 1-2 mm.

Nach 7 Monaten wurden die Stege ausgebaut, um die Stabilität der Behandlung bezüglich Mitteneinstellung für einen Zeitraum von ca. 6 Wochen zu überwachen, bevor das Herbstschanier definitiv entfernt wurde ( $\boldsymbol{}$ Abb. $\mathbf{5}$ und $\mathbf{6}$ ). Anschließend erfolgte das Bonding im Ober- und Unterkiefer mit einer konventionellen Multiband-Multibracketapparatur. Nach einem Jahr Multiband-Multbracket-Apparatur konnte eine Neutralokklusion im Bereich der 3er und 6 er bei physiologischem Overjet und Overbite eingestellt werden ( $\triangleright$ Abb. 7). Da sich der Durchbruch der Zähne 17 und 27 verzögerte, wurde die Lage und Position der Zähne mittels einer Panoramaschichtaufnahme (PSA) nochmals geprüft und kontrolliert. Die Auswertung ergab einen retinierten Zahn 17 und 27 mit Verlagerung von 27. Daher wurde das geplante Debonding verschoben und im Oberkiefer ein TPA mit posteriorem Ausleger eingesetzt. Die Zähne 17 und 27 wurden chirurgisch freigelegt und mit einem Knöpfchen mit Kette beklebt.

Im Unterkiefer wurde ein Lateleveler [21] zur Intrusion von 37 eingesetzt. Aktuell werden die 2. Molaren weiter eingestellt. Es 
konnten bereits Brackets auf die Zähne 17 und 27 geklebt werden. Leider war es aufgrund von Behandlungsverzögerungen im Rahmen der Coronapandemie noch nicht möglich den Patienten zu entbändern.

\section{Diskussion}

Zur Behandlung einer skelettalen Klasse-II-Malokklusion können sowohl herausnehmbare als auch festsitzende funktionskieferorthopädische Apparaturen zum Einsatz kommen [11, 12].

In dem vorliegenden Patientenfall wurde die Therapie aufgrund des Alters zunächt mit einer Vorschubdoppelplatte nach Sander durchgeführt. Die Vorschubdoppelplatte $[15,16]$ wird von Patienten in der Regel gut angenommen, da sie mit diesem Gerät im Mund recht gut sprechen können. Durch die unterschiedlichen Halteelemente ist es zudem möglich, eine relativ gute Verankerung auch im Wechselgebiss zu erreichen. Da die Konstruktion des Gerätes in 2 separaten Platten gleichzeitig ermöglicht neben der Bisslage und transversalen Dehnung auch Einzelzahnbewegungen im Ober- und Unterkiefer durchzuführen, konnten in relativ kurzer Zeit folgende Ziele erreicht werden: Korrektur der skelettalen Klasse II,
Korrektur des transversalen Defizits, Distalisieren der Oberkiefermolaren. Allerdings konnte die skelettale Mittenabweichung des Unterkiefers nicht vollständig korrigiert werden. Daher kam im nachfolgenden Schritt die Herbst-Apparatur zum Einsatz, da zu diesem Zeitpunkt der pubertäre Wachstumsgipfel bereits überschritten war. Sicherlich wäre der Therapiebeginn direkt mit der HerbstApparatur zu einem späteren Behandlungszeitpunkt (nach Überschreiten des pubertären Wachstumsgipfels) denkbar gewesen, allerdings sind hier oftmals die Wünsche und Erwartungen von Patient/Eltern und Kieferorthopäden nicht immer deckungsgleich.

In dem vorliegenden Patientenfall wurde die Latherognathie und damit die seitenungleiche Okklusion mittels eines asymmetrisch eingestellten Herbstschaniers und nachfolgenden klassischen Multibandbracketapparatur korrigiert. Untersuchungen haben gezeigt, dass eine Herbst-Apparatur besonders effektiv bei Patienten nach dem pubertären Wachstumsschub, also in der späten Adoleszenz (14-17 Jahre) ist und auch im jungen Erwachsenenalter stabile Veränderungen zeigt [14]. Im Gegensatz zu herausnehmbaren Geräten bietet die Herbst-Therapie auch allgemein den Vorteil, dass die Apparatur mit einer transversalen Gaumennahterweiterung (konservativ oder chirurgisch unterstützt) oder einer Lingualappa-
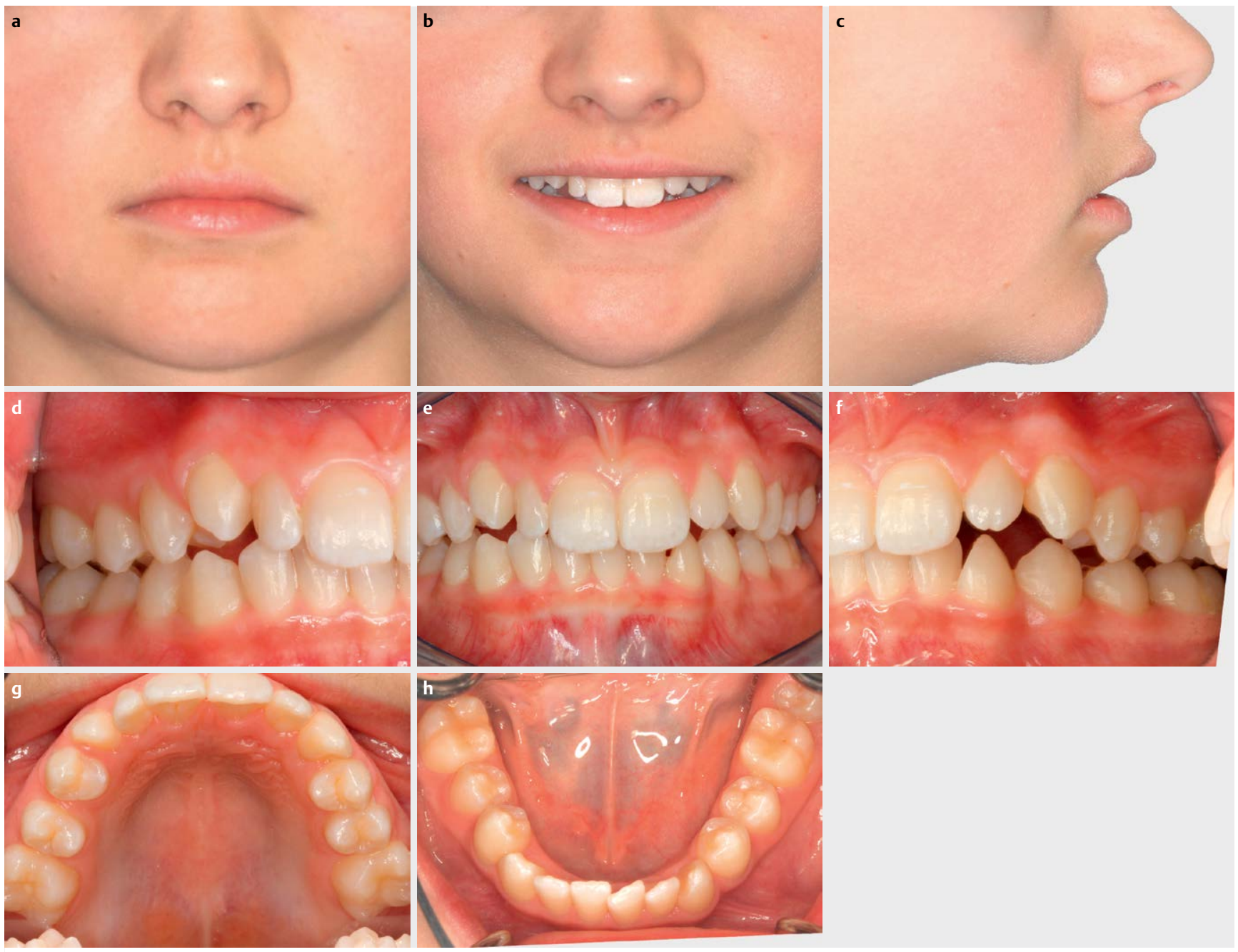

- Abb. 3 Klinischer Zwischenbefund a-c extraoral, d-h intraoral nach der Funktionskieferorthopädie (FKO). 
ratur kombinierbar ist und permanent, compliance-unabhängig über einen Zeitraum von mindestens 6 Monaten in beiden Kiefern verbleibt. Gerade weil die Apparatur während der aktiven Therapie 24 Stunden im Mund verbleibt, können neben den klassischen Befunden Mittenabweichungen und Distalbisslagen auch darüber hinaus pathologische Kiefergelenksbefunde (Diskusverlagerung) frühzeitig therapiert werden. Umgekehrt zeigen im Vergleich zur Literaturhäufigkeit von kraniomandibulären Dysfunktionen Langzeit-Nachuntersuchungen $[17,18]$ an erwachsenen Patienten nach Behandlungsende (bis zu 32 Jahren), dass die Herbst-Therapie grundsätzlich keine unerwünschten Nebenwirkungen im Bereich der Kiefergelenke verursacht.
Ein wesentlicher Vorteil der Herbst-Apparatur gegenüber herausnehmbaren Geräten ist auch, dass eine stärkere skelettale Wirkung erzielt werden kann, wobei der dentale Anteil grundsätzlich überwiegt (20\% skelettal vs. $80 \%$ dental, [19, 20]. Als klassische Indikation gilt die moderate skelettale Klasse II bei jugendlichen Patienten mit horizontalem bzw. neutralem Wachstumsmuster und retrudierten bzw. achsengerechten Unterkieferinzisiven [21]. Dass das Herbstschanier auch zur Korrektur von unilateralen Klasse-IIDysgnathien genutzt werden kann, konnte die Arbeitsgruppe Bock [22] in einer weiteren Studie zeigen. Die größeren Kräfte auf der stärker aktivierten Seite des Herbstschaniers führen der Arbeitgruppe Pancherz [23] zufolge zu einem ausgeprägterem kon-

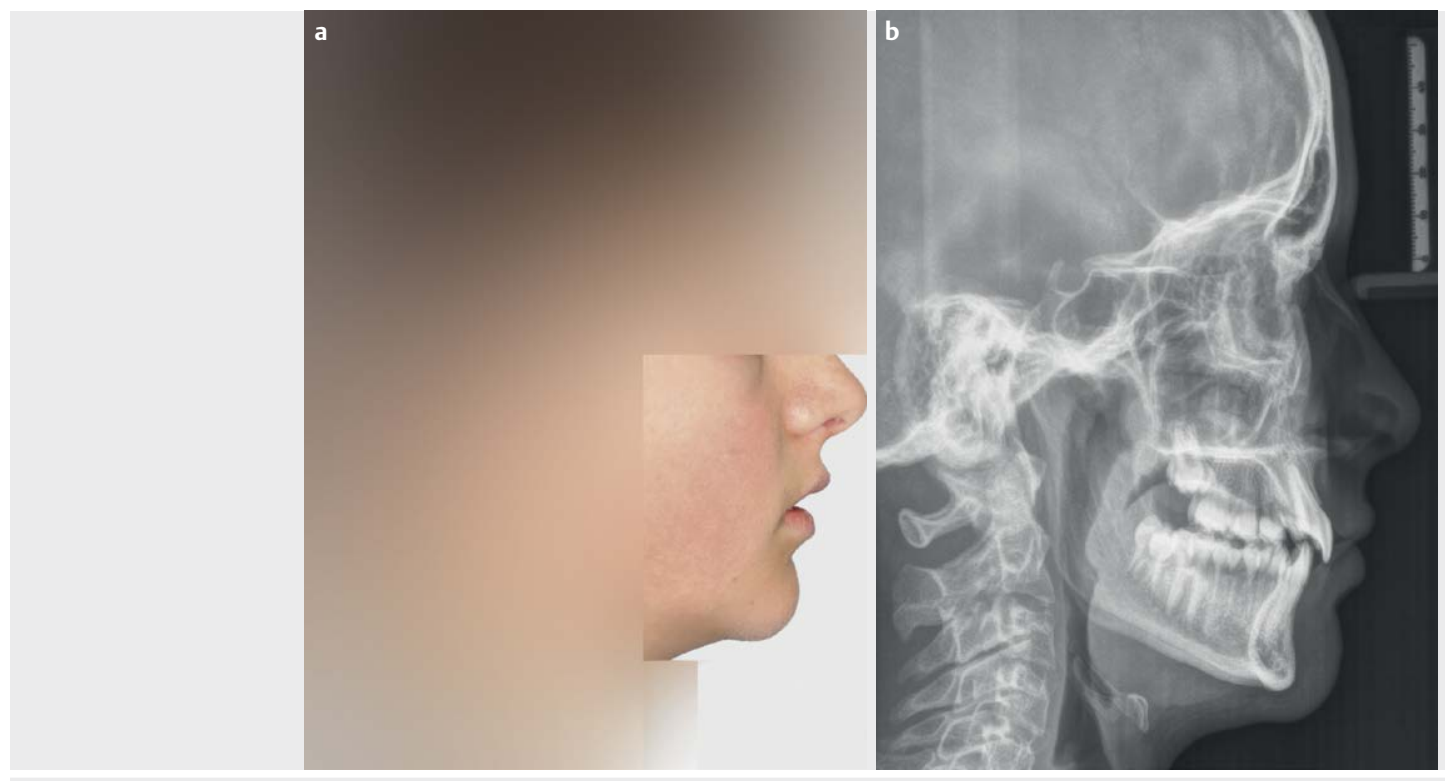

- Abb. 4 a Profilansicht sowie b Fernröntgenseitenbild nach der Funktionskieferorthopädie (FKO).
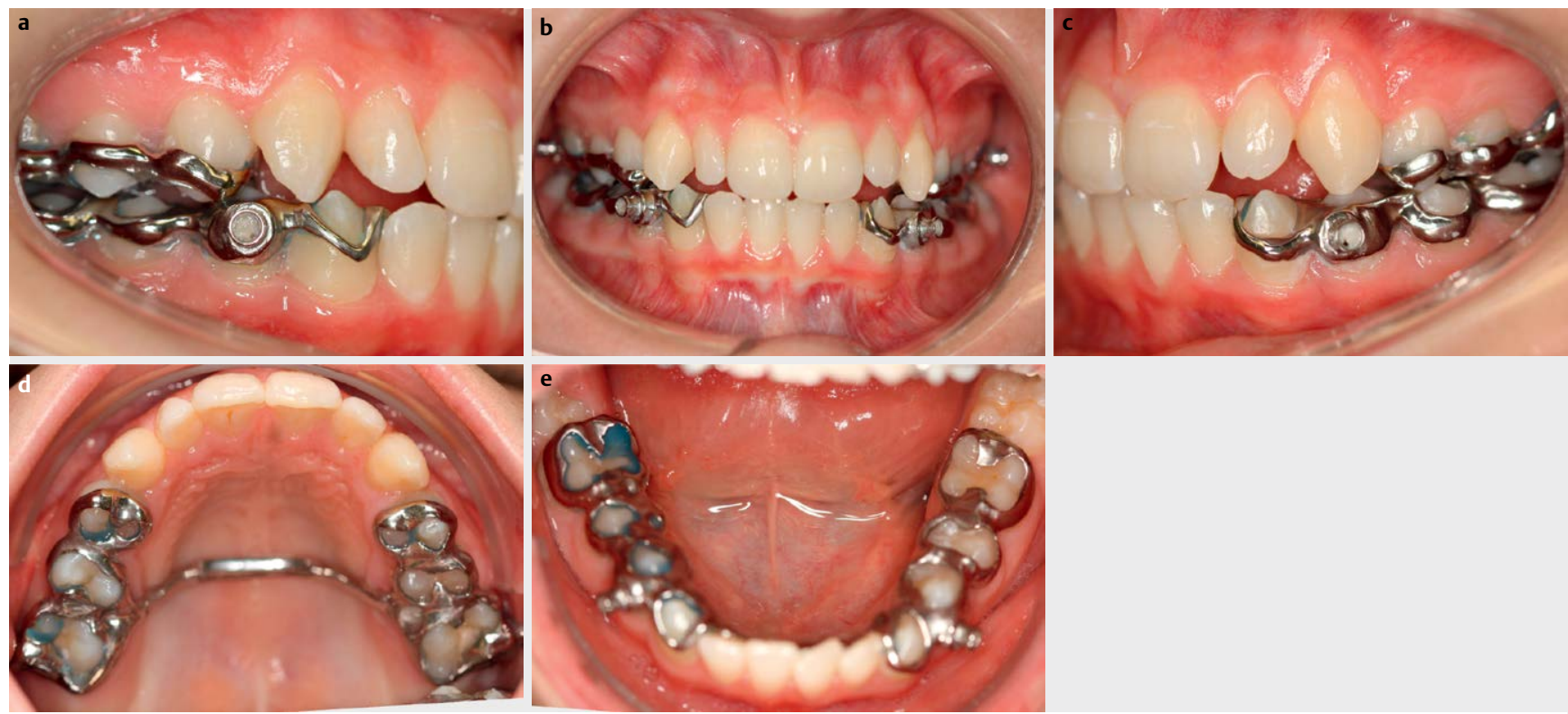

- Abb. 5 Eingesetzte Herbstapparatur in Situ. Die Stege wurden zur Kontrolle des erreichten Zustands ausgebaut. 

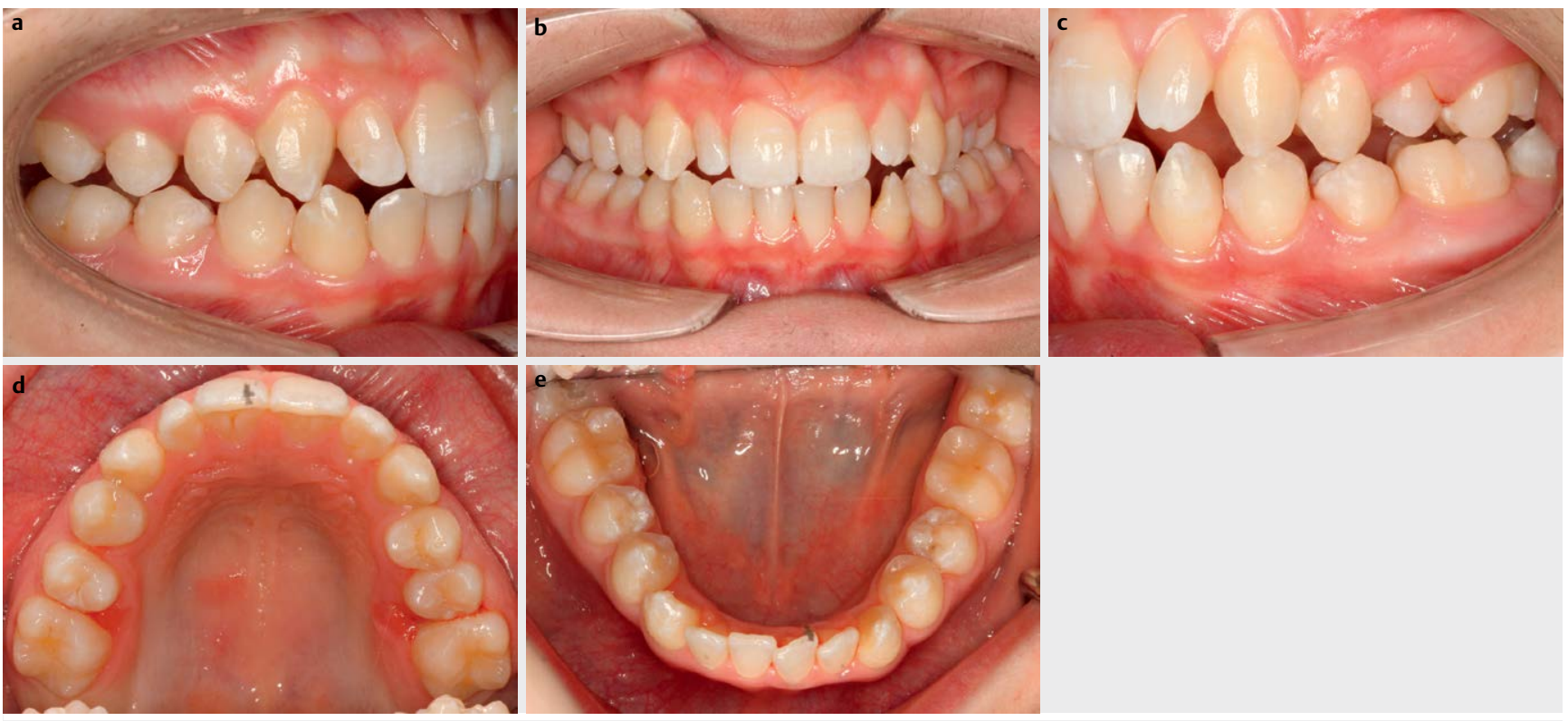

Abb. 6 Zwischendiagnostik nach der Entfernung der Herbstapparatur. Die Einstellung der Neutralokklusion rechst a, die Mitteneinstellung b sowie die Überkorrektur in eine leichte Mesialokklusion auf der linken Seite c.
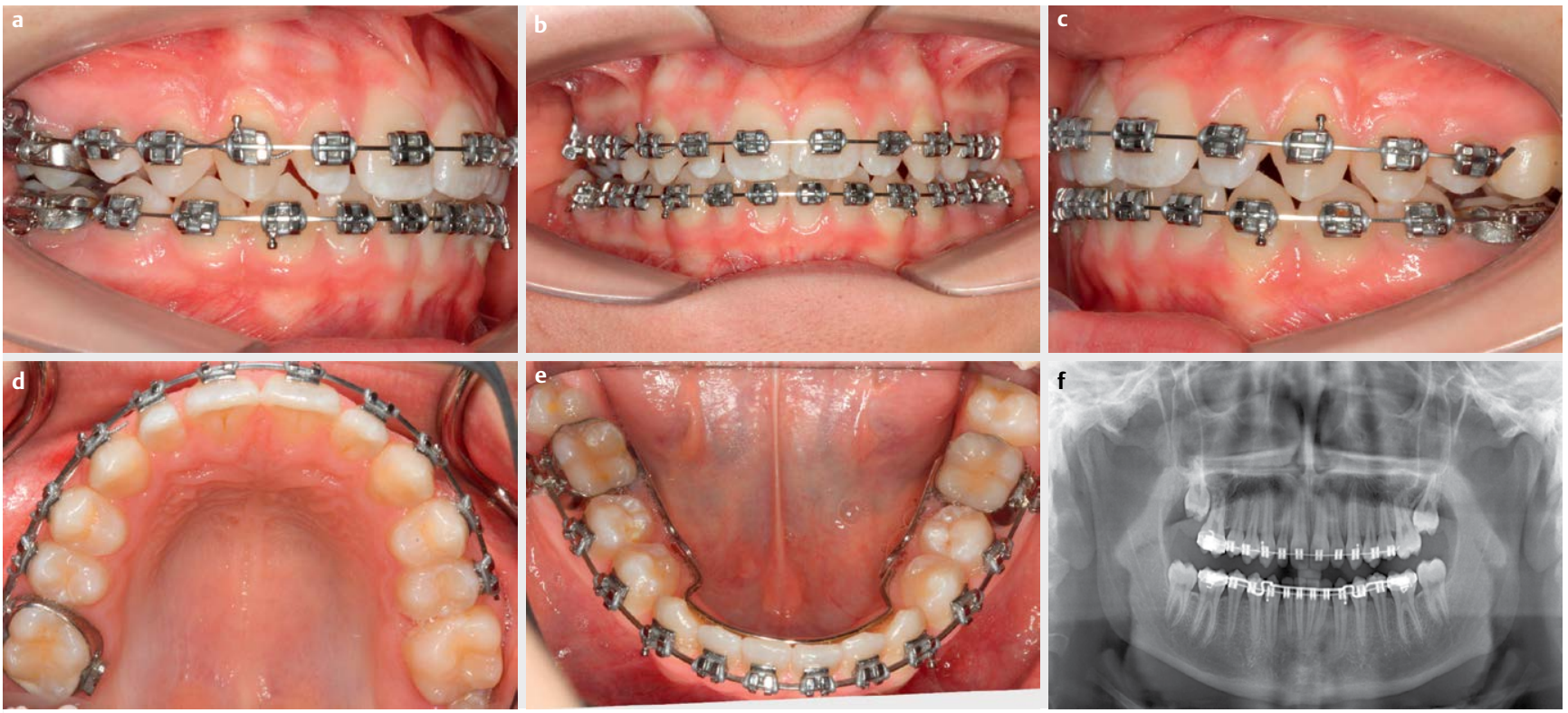

- Abb. 7 Zwischendiagnostik nach einem Jahr Therapie mittels Multiband-Multibracketapparatur. Zu sehen ist die Neutralokklusion im Bereich der 3er und 6er beidseits $\mathbf{a}$ und $\mathbf{c}$ bei physiologischem Overjet und Overbite b. Die Panoramaschichtaufnahme f zeigt die Retention der Zähne 17 und 27 sowie die Verlagerung von 27. Die Weisheitszähne sind nicht angelegt.

dylären Unterkieferwachstum sowie zu einer größeren kompensierenden Zahnbewegung (OK-Dentition nach posterior und UKDentiotion nach anterior). Als potenzielle Nebenwirkung konnten Bock und Mitarbeiter [22] auf der Seite mit der ursprünglichen Klasse-I-Verzahnung eine leichte Überkompensation beobachten, die zu einer Klasse-III-Tendenz führte. Diese Nebenwirkung zeigte sich auch bei unserem Patienten einseitig nach der Therapie der Klasse II auf der rechten Seite ( $\boldsymbol{A}$ Abb. $\mathbf{6 c})$. Diese konnte jedoch erfolgreich durch die nachfolgende Multiband-Multibracket-Apparatur und das Tragen von Klasse-III-Elastics links korrigiert werden
( $\bullet$ Abb. 7c). Wie bei der Vorschubdopppelplatte wird auch bei dem Herbstschanier ein „Headgear-Effekt“ in der Literatur beschrieben. Neben der Distalisation von bis zu 4,5 mm kann es auch zu einer Intrusion von bis zu 3,5 mm im Bereich der Molaren kommen. Daher kann der "Headgear-Effekt“" gut zur Platzbeschaffung im anterioren Oberkieferzahnbogen genutzt werden [24]. Allerdings könnte dieser Effekt im vorliegenden Patientenfall auch die mögliche Ursache für den Platzverlust im retromolaren Raum und damit für die Verlagerung/Retention der Zähne 17 und 27 gewesen sein. 


\section{Interessenkonflikt}

Die Autorinnen geben an, dass kein Interessenkonflikt besteht.

\section{Literatur}

[1] Ingervall B. Prevalence of dental and occlusal anomalies in Swedish conscripts. Acta Odontol Scand 1974; 32: 83-92

[2] Bažant V, Miksa J. Die Häufigkeit der Okklusionsanomalien in sagittaler Richtung in der Klassifikation nach Angle bei Erwachsenen. Fortschr Kieferorthop 1960; 21: 328-338

[3] Lippold C, van den Bos L, Hohoff A et al. Interdisciplinary study of orthopedic and orthodontic findings in pre-school infants. J Orofac Orthop 2003; 64: 330-340

[4] Brunelle JA, Bhat M, Lipton JA. Prevalence and distribution of selected occlusal characteristics in the US population, 1988-1991. J Dent Res 1996; 75: 706-713

[5] Vyslozil O, Jonke E. Kieferorthopädisch-antropometrische Vergleichsuntersuchung an 100 Jahre alten menschlichen Schädeln und österreichischen Bundesheersoldaten. Inf Orthod Kieferorthop 1994 26: 409-436

[6] Tschill P, Bacon W, Sonko A. Malocclusion in the deciduous dentition of Caucasian children. Eur J Orthod 1997; 19: 361-367

[7] Kloeppel J. Untersuchung über einige Entstehungsursachen des Distalbisses. Fortschr Kieferorthop 1961; 22: 388-394

[8] Grabowski R, Kundt G, Stahl F. Interrelation between occlusal findings and orofacial myofunctional status in primary and mixed dentition: Part III: Interrelation between malocclusions and orofacial dysfunctions. J Orofac Orthop 2007; 68: 462-476

[9] Harris EF, Johnson MG. Heritability of craniometric and occlusal variables: a longitudinal sib analysis. Am J Orthod Dentofacial Orthop 1991; 99: 258-268

[10] Zebrick B, Teeramongkolgul T, Nicot R et al. ACTN3 R577X genotypes associate with Class II and deepbite malocclusions. Am J Orthod Dentofacial Orthop 2014; 146: 603-611

[11] Koretsi V, Zymperdikas VF, Papageorgiou SN et al. Treatment effects of removable functional appliances in patients with Class II malocclusion: a systematic review and meta-analysis. Eur J Orthod 2015; 37 : 418-434
[12] Zymperdikas VF, Koretsi V, Papageorgiou SN et al. Treatment effects of fixed functional appliances in patients with Class II malocclusion: a systematic review and meta-analysis. Eur J Orthod 2016; 38: 113-126

[13] Kinzinger G, Czapka K, Ludwig B et al. Effects of fixed appliances in correcting Angle Class II on the depth of the posterior airway space: fMA vs. Herbst appliance-a retrospective cephalometric study. J Orofac Orthop 2011; 72: 301-320

[14] Bock NC, Ruf S. Class II division 2 treatment- does skeletal maturiy influence successand stability? J Orofac Orthop 2013; 74: 187-204

[15] Sander FG. Indikation für die Anwendung der Vorschubdoppelplatte. Prakt Kieferorthop 1988; 2: 209-222

[16] Sander FG, Wichelhaus A. Skelettale und dentale Veränderungen bei der Anwendug der Vorschubdoppelplatte. Fortschr Kieferorthop 1995; 56: 127-139

[17] Pancherz H, Salé H, Bjerklin K. Signs and symptoms of TM] disorders in adults after adolescscent Herbts therapy: a 6-year and 32-year radiograpfic and clinical follow-up study. Angle Orthod 2015; 85: 735-742

[18] Salé H, Bryndahl F, Isberg A. Temporomandibular koints in asymptomatic and symptomatic nonpatient volunteers: a prospective 15 -year folluw-up clinical and MR imaging study. Radiology 2013; 267: 183-194

[19] Pancherz H, Ruf S. The Herbst appliance: research-based clinical management. London: Quintessence Publishing; 2008

[20] Ruf S, Pancherz H. Dentoskeletal effects and facial profile changes in young adults treated with the Herbst appliance. Angle Orthod 1999; 69: 239-246

[21] Wichelhaus A, Eichenberg T, Günter A. Kieferorthopädie Therapie Band I. 2. unveränderte Auflage. Stuttgart: Thieme; 2017

[22] Bock NC, Reiser B, Ruf S. Class II subdivision treatment with the Herbst appliance. Angle Orthod 2013; 83: 327-333

[23] Pancherz H. Herbst-Behandlung der unilateralen Klasse-II-Dysgnathie. Kieferorthopädie 2016; 30: 235-242

[24] Pancherz H. „Headgear-Effekt” der Herbst-Apparatur. Kieferorthopädie 2020; 34: 153-164 